\title{
Ear Deformations Give Bats a Physical Mechanism for Fast Adaptation of Ultrasonic Beam Patterns
}

\author{
Li Gao, ${ }^{1,2}$ Sreenath Balakrishnan, ${ }^{2}$ Weikai He, ${ }^{1}$ Zhen Yan, ${ }^{1}$ and Rolf Müller ${ }^{2,1}$ \\ ${ }^{1}$ School of Physics, Shandong University, 27 Shanda South Road, Jinan, Shandong 250100, China \\ ${ }^{2}$ Department of Mechanical Engineering, Virginia Tech, Institute for Advanced Learning and Research, \\ 150 Slayton Avenue, Danville, Virginia 24540, USA \\ (Received 26 March 2011; published 14 November 2011)
}

\begin{abstract}
A large number of mammals, including humans, have intricate outer ear shapes that diffract incoming sound in a direction- and frequency-specific manner. Through this physical process, the outer ear shapes encode sound-source information into the sensory signals from each ear. Our results show that horseshoe bats could dynamically control these diffraction processes through fast nonrigid ear deformations. The bats' ear shapes can alter between extreme configurations in about $100 \mathrm{~ms}$ and thereby change their acoustic properties in ways that would suit different acoustic sensing tasks.
\end{abstract}

DOI: 10.1103/PhysRevLett.107.214301

PACS numbers: $43.80 . \mathrm{Ka}$

Bats occupy a wide variety of sensory-ecological niches [1-3]. Some of this diversity has likely been facilitated by adaptations in properties of the biosonar system such as the waveform of the emitted pulses $[4,5]$ or the beam pattern [6]. Based on the duty cycles of their pulse trains, bats are frequently grouped into high- (HDC) and low-duty cycle (LDC) species $[7,8]$. In the biosonar pulses of LDC bats, frequency changes continuously over time, whereas it stays constant for portions of the pulses of HDC species.

Beam patterns for sound reception describe the distribution of receiver sensitivity over direction at a given frequency. The biosonar beam patterns of several species of bats have been characterized with a variety of experimental and numerical methods already [6,9-12]. A comparison of numerical beam pattern estimates from a set containing different species of HDC and LDC bats (Fig. 1) indicates the presence of categorical differences between these two sensory-ecological groups: In HDC bats, beam patterns (Fig. 1, top row) tend to be simple and dominated by a single global maximum (main lobe). Additional local sensitivity maxima (side lobes) tend to be few and of much lower amplitude than the main lobe. In contrast to this, the beam patterns of LDC bats (Fig. 1, bottom row) tend to have prominent side lobes that change direction in a frequency-dependent fashion. Previously published bat biosonar beam patterns of LDC [13-16] and HDC bats [17] support the existence of these differences. The beam patterns of HDC bats are well suited for on-axis target detection or identification but are not well adapted for estimating target direction based on spectral signatures.

HDC bats-and horseshoe bats in particular-have long been known for their conspicuous pinna movements $[18,19]$, and early anatomical work [18] has demonstrated the presence of specialized muscular actuation mechanisms that enable them. Experimental results have demonstrated that immobilizing the pinnae of these bats degrades target localization in the vertical direction [20]. The target direction cues generated by rigid rotation of a receiver and its static beam pattern in target localization have also been analyzed already [21]. Rotations of an otherwise unchanged beam pattern have also been suggested as an approximation to the effects of ear movements in cats [22]. Whereas the rigid pinna rotations can only reorient an otherwise unchanged beam pattern, a nonrigid change in pinna shape could potentially alter the geometry of the beam pattern and thereby provide additional degrees of freedom for encoding relevant information into the received signals.

In order to investigate whether bats can carry out nonrigid pinna deformations, we have built time-varying, complete 3D digital models of the pinnae of behaving greater horseshoe bats, Rhinolophus ferrumequinum. To accomplish this, we have combined high-speed computer vision, high-resolution computer tomography, and numerical mechanical deformable model formulations [23].

Using these methods, cyclical nonrigid changes in pinna shape were observed: During each cycle, the pinna transitioned from an upright position to a bent position and back. When bending, the tip of the pinna moved down, outwards and backwards relative to the head. The lateral portions of the pinna moved in opposite directions with the frontal portion moving distally and the caudal portion moving proximally (Fig. 2). Movements conforming with this pattern were observed in all 13 individuals of which highspeed video recordings were obtained. The maximum deformation component of the displacement was typically more than $4 \mathrm{~mm}$ which corresponds to $\sim 15 \%$ of the pinna height and is similar in size to the wavelengths in the strongest harmonic $(4.3-5.7 \mathrm{~mm})$ of the biosonar pulse. Hence, the observed deformations were large enough to effect considerable change in the phase relationship between wave field contributions from different parts of the pinna surface and alter the beam pattern substantially. The movements occurred on a time scale similar to the length 

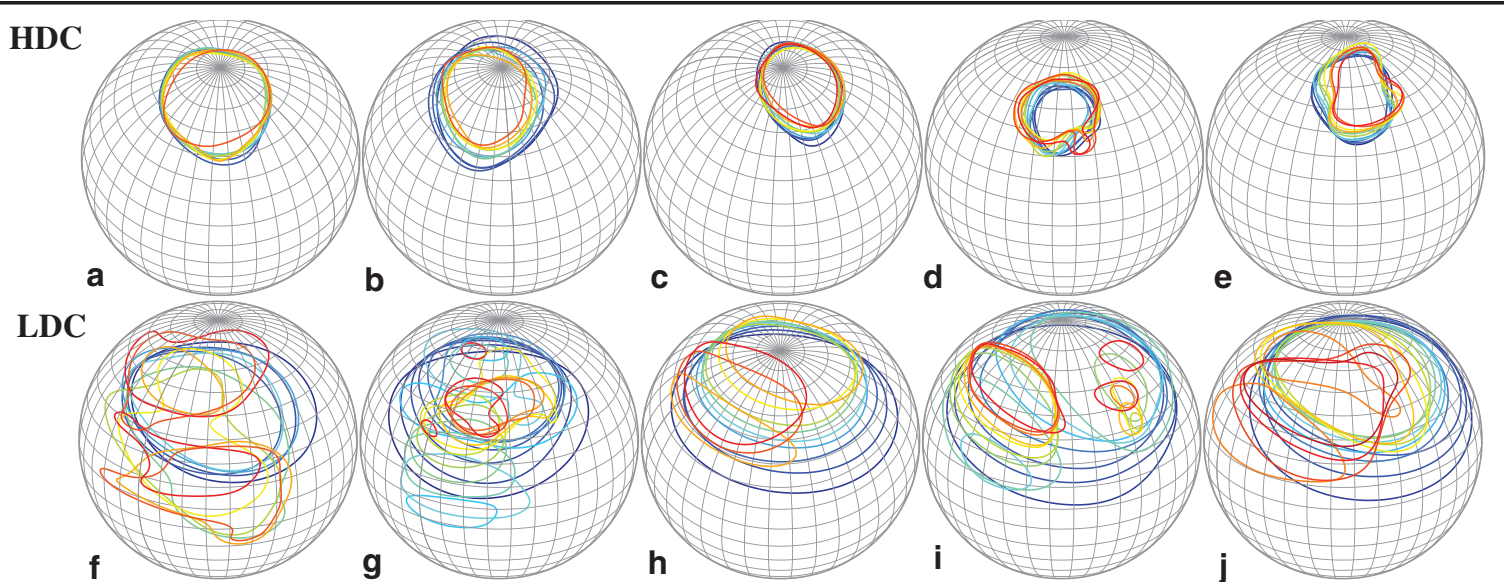

FIG. 1 (color). Beam pattern examples from HDC bats (top row) and LDC bats (bottom row). (a) Stoliczka's trident bat (Aselliscus stoliczkanus, 104-120 kHz in steps of $2 \mathrm{kHz}$ ), (b) Pearson's horseshoe bat (Rhinolophus pearsonii, 44-62 kHz in steps of $2 \mathrm{kHz}$ ), (c) Least horseshoe bat (Rhinolophus pusillus, $90-110 \mathrm{kHz}$ in steps of $2 \mathrm{kHz}$ ), (d) Pomona roundleaf bat (Hipposideros pomona, 100-126 kHz in steps of $2 \mathrm{kHz}$ ), (e) Pratt's roundleaf bat (Hipposideros pratti, 50-70 kHz in steps of $2 \mathrm{kHz}$ ), (f) Common bent-wing bat (Miniopterus schreibersii, 47-87 kHz in steps of $4 \mathrm{kHz}$ ), (g) Round-eared tube-nosed bat (Murina cyclotis, 40-176 kHz in steps of $8 \mathrm{kHz}$ ), (h) Rickett's big-footed bat (Myotis ricketti, 30-70 kHz in steps of $4 \mathrm{kHz}$ ), (i) Pipistrelle (Pipistrellus sp., 35-100 kHz in steps of $5 \mathrm{kHz}$ ), (j) Lesser bamboo bat (Tylonycteris pachypus, $50-120 \mathrm{kHz}$ in steps of $5 \mathrm{kHz}$ ). For each frequency, a single contour line is drawn at a gain level of $-6 \mathrm{~dB}$. Frequencies are coded by color from blue for the lowest frequency to red for the highest frequency analyzed.

of the pulse in these animals $(\sim 50 \mathrm{~ms},[24])$ with entire cycles often being completed in less than $200 \mathrm{~ms}$ (sometimes cycles last considerably longer, e.g., Fig. 2).

Efficient numerical methods for estimating beam patterns from digital shape models have been used to analyze the ear geometry from different individuals, stages of the
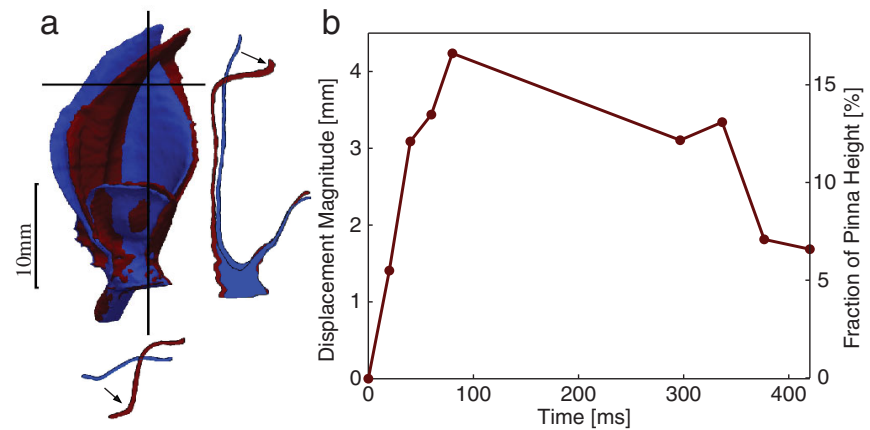

FIG. 2 (color). Example of a typical nonrigid pinna deformation geometry in the greater horseshoe bat. (a) Upright (blue) and bent (red) state of the pinna. Black lines mark the location of the cutting planes. The arrows in the section views show the direction of movement of various portions of the pinna. (b) Corresponding change in the magnitude of the displacement as a function of time during a deformation cycle. The displacement magnitude shown is the maximum over the displacements of all landmarks. Landmark displacements and time were measured from the most upright state of the pinna. The displacement magnitude is given in absolute terms (left axis) and as a fraction of pinna height (right axis). This example is only typical in geometry of the deformation, not in the duration of the deformation cycle (which is considerably longer than average). deformation cycle, and ultrasonic frequencies [23,25]. Each deformation cycle was sampled at nine (eight in two cases) instants in time that were representative of the different cycle stages. For each instant, the beam patterns of the respective pinna geometries were obtained for sound frequencies from 60 to $80 \mathrm{kHz}$ at $5 \mathrm{kHz}$ intervals. The sampled frequency range covers the observed band of the strongest harmonic of the individuals' biosonar $(\sim 60$ to $\sim 77 \mathrm{kHz}$ ).

Systematic changes in the beam patterns were seen as the pinnae bent and straightened again. These changes resulted in quantitative as well as qualitative beam pattern differences between the upright and bent pinna geometries that were consistent across all recordings: For the upright position of the pinna, the side lobes of the beam pattern were weak compared to the main lobe (as much as $-15 \mathrm{~dB}$ difference between maxima). As the pinna deformed, the sensitivity in the side lobes increased significantly relative to the main lobe (Fig. 3). The relative contributions of a main lobe or a set of side lobes to the overall sensitivity of the pinna were quantified by integrating the sensitivity gain over the solid angle of the respective lobe or lobes ("total sensitivity," see Fig. 4). Regardless of bending state, the beam patterns always contained a strong main lobe (Fig. 3) that contributed approximately the same amount of integrated sensitivity to the normalized beam pattern. In contrast to this, the contribution of the side lobes to the total sensitivity in the normalized beam pattern increased dramatically in the bent shape configuration of the pinna [Fig. 4(a)]. At maximum, the side lobe sensitivity was about 5 times larger in the bent than in the upright pinna [Fig. 4(a)]. In some cases, the sensitivity maxima of a side 


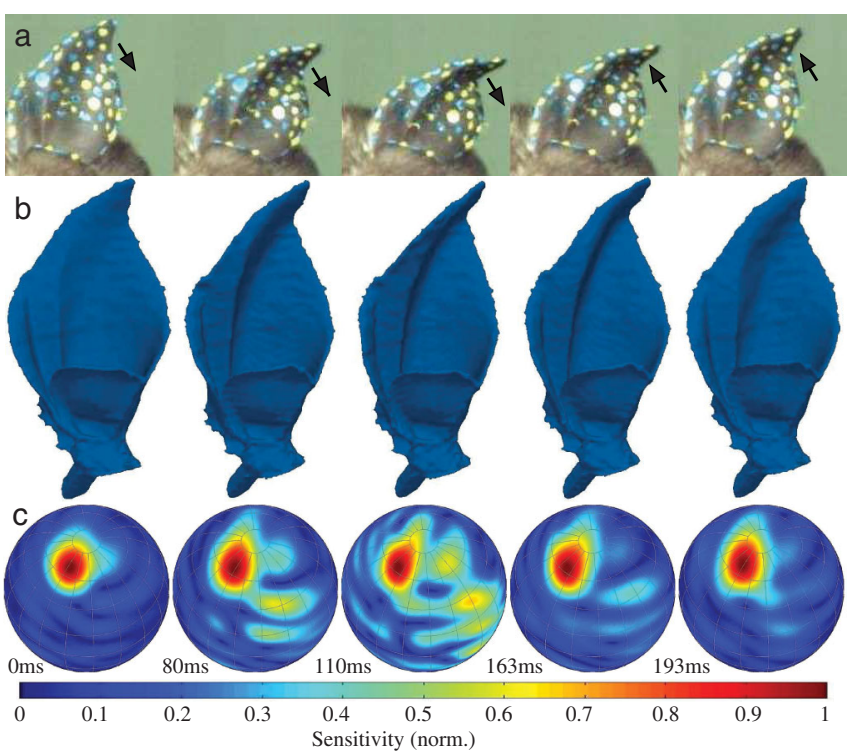

FIG. 3 (color). Beam pattern changes with pinna deformation. (a) Image sequence of the pinna deformations captured by a high-speed video camera with the arrows indicating the pinna tip's approximate direction of motion in each frame. (b) Renderings of the complete digital model of the pinna at the same time instants as the video frames. (c) The normalized far-field directivity gains in different directions (beam patterns), at a resolution of $1^{\circ}$ in azimuth and elevation, computed for each of the pinna geometries at a sound frequency of $75 \mathrm{kHz}$. The orientation of the beam pattern matches that of the shape renderings approximately; i.e., the central axis of the pinna cone points towards the North Pole.

lobe even surpassed the respective main lobes to become the global sensitivity maximum. Investigation of the raw (not normalized) beam pattern data revealed that the changes in the relative weight of main lobe to side lobe was primarily due to an increased sensitivity in the side lobe; the gain of the main lobe changed comparatively little during deformation of the pinnae. When the pinnae returned from their bent state to an upright position at the end of the deformation cycle, the beam patterns also reverted to their previous configuration [Figs. 3 and 4(a)].

To characterize the changes in the main and side lobes over frequency, an overlap between the solid angles enclosed by $-3 \mathrm{~dB}$ isogain contours was computed as a similarity measure. It was used to compare the position and geometry of the same lobes across different frequencies. The average of the overlap between the lobes of the four pairs of neighboring frequencies computed was then taken as a single measure of change with frequency. Using this similarity measure, it was confirmed that the main lobes changed little across frequency [Fig. 4(b)]. As was the case for the lobe strength, the behavior of the side lobes differed from that of the main lobes. For the majority of the side lobes, position and shape depended strongly on frequency. As a result, the side lobes at neighboring
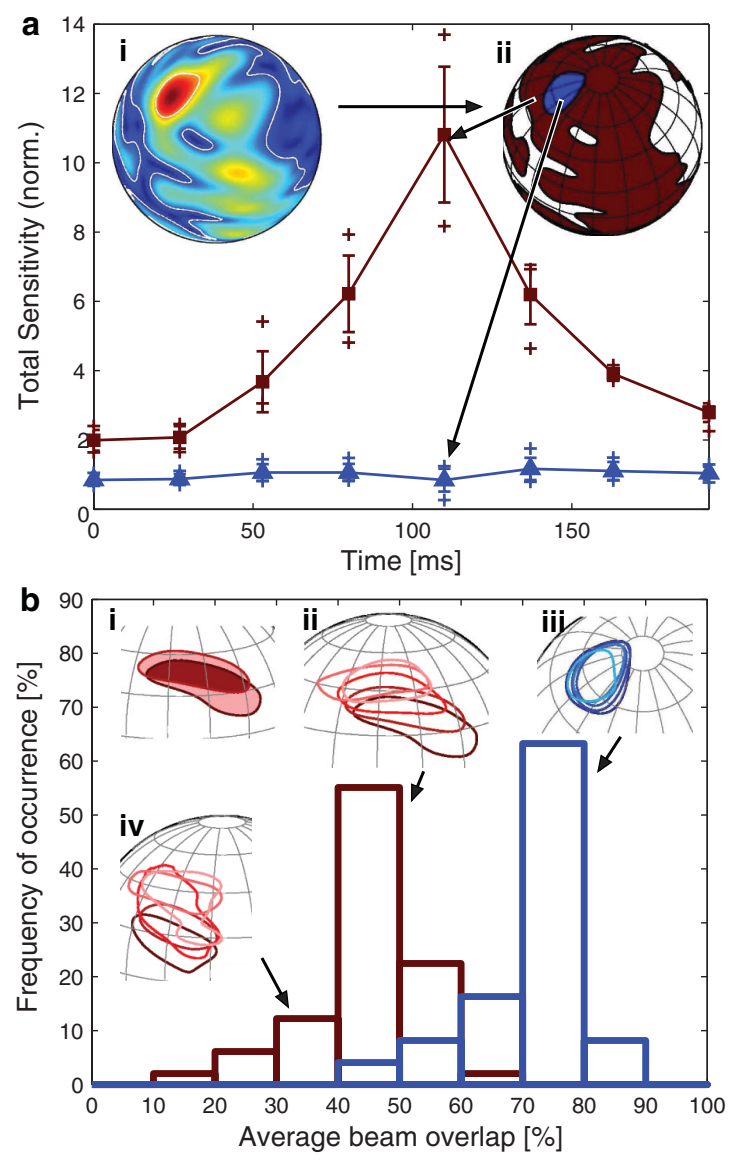

FIG. 4 (color). Beam pattern analysis. (a) Change in total sensitivity of the main lobe $(\boldsymbol{\Delta})$ and side lobes ( $\boldsymbol{\square})$ over a deformation cycle: average $(\boldsymbol{\Lambda}, \boldsymbol{\square})$, standard deviation (error bars), and extreme values $(+)$ over frequency $(60-80 \mathrm{kHz}$ in $5 \mathrm{kHz}$ intervals). The main lobe is defined to cover all directions inside a $-3 \mathrm{~dB}$ contour that contains the global maximum [inner white contour in inset (i), solid blue in inset (ii)]. The remaining directions are allocated to the side lobes [solid red in inset (ii)] as long as their gain values do not fall below $-15 \mathrm{~dB}$ of the global maximum. The total sensitivity of a lobe is defined as the integral of directivity gain over the lobe's area. Values shown are normalized with respect to the mean total sensitivity value of the main lobe (taken over all pinna geometries and frequencies). (b) Comparison of the frequency dependence of main lobe and side lobe: Histograms of the average beam overlap of the main lobes (blue) and side lobes (red) in beam patterns for adjacent frequencies. The areas of main lobes and side lobes were defined by $-3 \mathrm{~dB}$ contours that include the lobe maxima [insets (i)-(iv)]. Only the strongest side lobe was used in this analysis. Beam overlap was used as a measure of change in beam position and shape over frequency. It was defined as the ratio of the intersection to the union of the areas of one lobe for two adjacent frequencies [inset (i)]. Average beam overlap was obtained by taking the mean over all neighboring frequencies. Only pinna geometries with significant side lobes (average total sensitivity in the side lobes greater than 3 times the average total sensitivity in the main lobe; 49 shapes met this criterion) were used in this analysis. Insets (ii)-(iv) show example lobe areas as a function of frequency where frequency is color coded such that highest frequency is the lightest. 


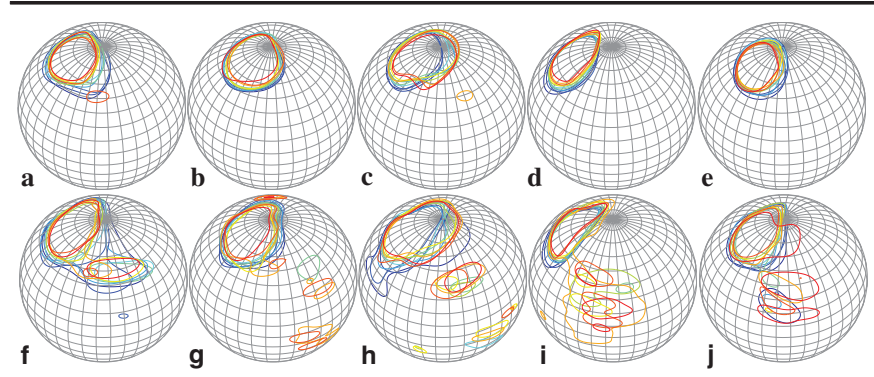

FIG. 5 (color). Comparison of example beam patterns of pinna shapes from greater horseshoe bats in an upright (top row) and a bent pose (bottom row). (a)-(e) Five different individuals of Greater horseshoe bats (Rhinolophus ferrumequinum), upright in vivo shapes (60-80 kHz in steps of $2 \mathrm{kHz}$ ), and (f)-(j) in vivo bent shapes from the same Greater horseshoe bat individuals using the same frequencies.

frequencies overlapped much less across frequency than those of the main lobes [Fig. 4(b), $p<0.1 \%$, two-sample Kolmogorov-Smirnov test]. The frequency dependence of the side lobes manifested itself either in orderly patterns of change, e.g., in lobe direction [Fig. 4(b)(iii)] or in less easily comprehensible changes that affected either lobe direction, lobe shape, or both [Fig. 4(b)(iv)].

All changes in the beam pattern as a function of ear deformation described here were seen in a qualitatively similar fashion across all studied frequencies. There were no systematic differences between the behavior of beam patterns with deformation at different frequencies. In particular, no significant differences between the sub-bands of the frequency-modulated and the constant-frequency portions of the biosonar pulses were found.

Different beam patterns have different merits and are hence better suited for some estimation tasks than for others. For example, beam patterns that concentrate their sensitivity for all frequencies in a single direction are well suited for detection of weak signals coming from this direction. They also favor the identification of sound sources from the spectral signature of the received echoes [26]. In this case, the concentration of sensitivity maximizes the signal-to-noise ratio which also helps identification. Furthermore, orienting the main lobes across all frequencies in the same direction results in a flat transfer function for signals from sources in that direction. This facilitates spectral identification because the spectral signature of the target is not perturbed during echo reception. The beam patterns of all the upright pinnae from the behavioral experiments as well as those of the static shapes from other HDC bat species analyzed (examples shown in Fig. 1 top row), fall under this category. Accurate monaural target localization imposes requirements on the beam pattern that may conflict with detection and identification [26]: It is favored by beam patterns with frequencydependent change in the direction of the lobes that encode information on target direction into the echo's spectral profile [11]. Accurate monaural direction finding requires large changes in the beam gain with direction and frequency [Figs. 4(b)(ii) and 4(b)(iv)], but not necessarily in an orderly or easily comprehensible fashion. The beam pattern estimates obtained for the pinnae of LDC bat species (examples in Fig. 1 bottom row) fall into this category. The beam pattern estimates for the bent pinnae in the behavioral experiments also show the same qualitative features (Fig. 5).

Our findings suggest that horseshoe bats could use nonrigid deformations of their ears as a physical mechanism to switch between beam patterns with different qualities and hence different functional roles. How these different patterns could be integrated into the animals' biosonar system remains an open question: Even though attempts to correlate biosonar emissions and ear movements have been made in early studies $[19,27]$, it remains to be determined what the time relationships between ear deformations, received echoes, and informational needs of the animals are. Since the time durations of the deformations and the emitted pulse are fairly similar, transitions between these ear shapes with different acoustic functions could happen either between successive echoes or within a single echo. Further behavioral experiments with bats are needed to determine if and how the animals make use of the dynamic adaptation substrate that the nonrigid pinna deformations provide.

This work was supported by the National Natural Science Foundation of China (Projects No. 10774092 and No. 11074149), the Shandong University Independent Innovation Foundation (Project No. 2010JC001), the Shandong Taishan Scholar Fund, and the China Scholarship Council.

[1] G. Neuweiler, Trends Ecol. Evol. 4, 160 (1989).

[2] J.E. Hill and J.D. Smith, Bats: A Natural History (University of Texas, Austin, 1992).

[3] H.-U. Schnitzler and E. Kalko, in Bat Biology and Conservation, edited by T. Kunz and P. Racey (Smithsonian Institution, Washington D.C., 1998), pp. 183-196.

[4] R. A. Altes and D. P. Skinner, J. Acoust. Soc. Am. 61, 1019 (1977).

[5] J. A. Simmons and R. A. Stein, J. Comp. Physiol. 135, 61 1980).

[6] R. Müller, J. Acoust. Soc. Am. 128, 1414 (2010).

[7] G. Jones and E. C. Teeling, Trends Ecol. Evol. 21, 149 (2006).

[8] L. Lazure and M. Fenton, J. Exp. Biol. 214, 1131 (2011).

[9] Q. Zhuang and R. Müller, Phys. Rev. Lett. 97, 218701 (2006).

[10] Z. Zhang, S. N. Truong, and R. Müller, Phys. Rev. Lett. 103, 038701 (2009).

[11] R. Müller, H. Lu, and J. R. Buck, Phys. Rev. Lett. 100, 108701 (2008). 
[12] J. Reijniers, D. Vanderelst, and H. Peremans, Phys. Rev. Lett. 105, 148701 (2010).

[13] R. Müller, J. Acoust. Soc. Am. 116, 3701 (2004).

[14] J. M. Wotton, T. Haresign, and J. A. Simmons, J. Acoust. Soc. Am. 98, 1423 (1995).

[15] M. Aytekin, E. Grassi, M. Sahota, and C.F. Moss, J. Acoust. Soc. Am. 116, 3594 (2004).

[16] R. Müller, H. Lu, S. Zhang, and H. Peremans, J. Acoust. Soc. Am. 119, 4083 (2006).

[17] U. Firzlaff and G. Schuller, Hear. Res. 197, 74 (2004).

[18] H. Schneider and F. Möhres, Z. Vgl. Physiol. 44, 1 (1960).

[19] D. Griffin, D. Dunning, D. Cahlander, and F. Webster, Nature (London) 196, 1185 (1962).

[20] J. Mogdans, J. Ostwald, and H.-U. Schnitzler, J. Acoust. Soc. Am. 84, 1676 (1988).
[21] V. A. Walker, H. Peremans, and J. C. T. Hallam, J. Acoust. Soc. Am. 104, 569 (1998).

[22] E. Young, J. Rice, and S. Tong, J. Acoust. Soc. Am. 99, 3064 (1996).

[23] See supplemental Material at http://link.aps.org/ supplemental/10.1103/PhysRevLett.107.214301 for details on the deformable model formulation and numerical acoustic methods.

[24] B. Tian and H. Schnitzler, J. Acoust. Soc. Am. 101, 2347 (1997).

[25] Q. Zhuang and R. Müller, Phys. Rev. E 76, 051902 (2007).

[26] K. R. Rao and J. Ben-Arie, IEEE Trans. Biomed. Eng. 43, 1093 (1996).

[27] J. D. Pye and L.H. Roberts, Nature (London) 225, 285 (1970). 